\title{
Sôbre uma. Fórmula para o Cálculo da Dose Mais Economica de Adubo
}

\author{
F. PIMENTEL GOMFS
}

CLOVIS POMPILIO DE ABREU

E. S. A. "Luiz de Queiroz" 


\section{INTRODUÇÃO}

CAREY e ROBINSON (1953) introduziram nova fórmula para o cálculo da dose $\mathrm{x}^{*}$ econômicamente aconselhável. Tal fórmula foi logo adotada por PIMENTEL GOMES (1957) e é, aliạs, uma modificação de uma das fórmulas de PIMENTEL GOMES (1953) e um caso particular de uma fórmula de PIMENTEL GOMES E MALAVOLTA (1949). assim:

$$
x^{*}=(1 / c) \log \frac{c x_{u} L 10}{1-10^{-c x_{u}}}+(1 / c) \log \left(w u / x_{u} t\right),
$$

onde $c$ é o coeficiente de eficácia de Mitscherlich, $\mathrm{x}_{\mathrm{u}}$ é uma dose de nutriente tomada como padrão, L 10 é o logarítmo neperiano de 10, $u$ é a resposta padrão, isto é, o aumento de produção do produto agrícola causado pela dose padrão $\mathrm{x}_{\mathrm{u}}$ do nutriente, $w$ é o preço da unidade do produto agrícola, no campo, e $t$ é o custo da unidade de nutriente.

Uma vantagem dessa fórmula é que não depende de $A$, produção máxima teórica possível, que não é dada diretamente pelos resultados experimentais. Mas uma vantagem muito maior, inédita, que demonstraremos adiante, é que o primeiro têrmo

$$
K=(1 / c) \log \frac{c x_{\mathfrak{u}} \mathrm{L} 10}{1-10^{-c \mathbf{x}_{\mathbf{u}}}}
$$

é praticamente independente de $c$, sendo muito aproximadamente igual à metade da dose padrão, isto é, equivalente a $(1 / 2) x_{u}$.

Tal propriedade permite fácil aplicação da fórmula em qualquer caso em que conheçamos o aumento de produção $u$ causado por uma dose padrão arbitrária $x_{u}$, e a facilidade se torna ainda maior pelo uso de tabelas incluidas neste trabalho. 
2. DEMONSTRAÇÃO DE UMA PROPRIEDADE DA FÓRMULA

Pássamos a demonstrar que o têrmo

$$
\mathrm{K}=(1 / \mathrm{c}) \log \frac{\mathrm{c} \mathrm{x}_{\mathrm{u}} \mathrm{L} 10}{1-10^{-\mathrm{cx}} \mathrm{u}}
$$

é pràticamente independente de $c$ e aproximadamente equi valente a $(1 / 2) \mathbf{x}_{\mathbf{u}}$.

Temos, com grande aproximação, para valores não muitc elevados de $\mathbf{X}$ :

$$
\begin{aligned}
& \mathrm{e}^{\mathrm{x}}=1+\mathrm{X}+(1 / 2) \mathrm{x}^{2}, \log 0 \\
& 1-10^{-\mathrm{cxu}}=1-\mathrm{e}^{\mathrm{cx_{ \textrm {u } }}} \quad \mathrm{L} 10=\mathrm{cx}_{\mathrm{u}} \text { L } 10-\frac{1}{2}\left(\mathrm{cx}_{\mathrm{u}} \mathrm{L} 10\right)^{2}
\end{aligned}
$$

Obtemos, pois,

$$
\begin{aligned}
K & =(1 / c) \log \frac{\mathrm{cx}_{\mathrm{u}} \mathrm{L} 10}{\mathrm{cx}_{\mathrm{u}} \mathrm{L} 10-(1 / 2)\left(\mathrm{cx}_{\mathrm{u}} \mathrm{L} 10\right)^{2}} \\
& =(1 / \mathrm{c}) \log \frac{1}{1-(1 / 2) \mathrm{cx}_{\mathrm{u}} \mathrm{L} 10}
\end{aligned}
$$

Mas, temos ainda com boa aproximação

$$
10-(1 / 2) c x_{\mathrm{u}}=\mathrm{e}^{-(1 / 2) c \mathrm{x}_{\mathrm{u}} \mathrm{L} 10}=1-(1 / 2) c \mathrm{x}_{\mathrm{u}} \mathrm{L} 10,
$$

portanto

$$
\begin{aligned}
\mathrm{K} & =(1 / \mathrm{c}) \log \frac{1}{10-(1 / 2) c \mathrm{x}_{\mathrm{u}}} \\
& =(1 / \mathrm{c})(1 / 2) \mathrm{c} \mathrm{x}_{\mathrm{u}} \\
& =(1 / 2) \mathrm{x}_{\mathrm{u}}
\end{aligned}
$$

Mostremos, agora, com exemplos, que a aproximação é realmente notável, mais do que suficiente para fins práticos.

Sabe-se que, nos casos de $\mathrm{N}, \mathrm{P}_{2} \mathrm{O}_{5}$ e $\mathrm{K}_{2} \mathrm{O}$, tomado como unidade o quintal métrico, $\mathrm{o}$ valor de $c$ está quase sempre entre 0,20 e 1,00. 
A dose padrão $\mathrm{x}_{\mathrm{u}}$ é arbitrária, mas em geral não deverá ser muito baixa, nem excessivamente elevada. Se adotarmos, a título de exemplo, a dose padrão de 60 kilos/hectares, pràticamente igual à usada por HODNETT ( $56 \mathrm{~kg} / \mathrm{ha}$. ou um quintal longo por acre), teremos os valores seguintes para $\mathrm{K}$.

\begin{tabular}{c|c|c}
\hline $\begin{array}{c}\text { Valores de c } \\
\text { (ha/quintal métrico) }\end{array}$ & $\begin{array}{c}\text { Valores de } \mathrm{K} \\
(\mathrm{kg} / \mathrm{ha})\end{array}$ & $\begin{array}{c}(1 / 2) \mathrm{x}_{\mathrm{u}} \\
(\mathrm{kg} / \mathrm{ha})\end{array}$ \\
\hline 0,20 & 29,4 & 30,0 \\
0,50 & 28,3 & 30,0 \\
0,90 & 26,9 & 30,0 \\
1,00 & 26,6 & 30,0 \\
\hline
\end{tabular}

Para o caso do estêrco, dados de CROWTHER e YATES (1941) e de CAREY e ROBINSON (1953), bem como determinação feita por F. Pimentel Gomes com dados inéditos de E. J. Kiehl e Cyro Marcondes Cesar, demonstraram que o valor de $c$ é aproximadamente 0,018 , quando a dose de estêrco é expressa em toneladas por hectare. Se adotarmos, então, como dose padrão, $\mathbf{x}_{\mathbf{u}}=10 \mathrm{t} / \mathrm{ha}$, teremos

$$
K=4,83 \mathrm{t} / \mathrm{ha} \text {, quando }(1 / 2) \mathrm{x}_{\mathrm{u}}=5,00 \mathrm{t} / \mathrm{ha} .
$$

Em qualquer caso, temos, pois, com grande aproximação:

$$
x^{*}=(1 / 2) x_{u}+\frac{1}{c} \log \left(w u / x_{u} t\right)
$$

\section{TABELAS PARA APLICAÇÃO DA FORMULA}

Na última fórmula, wi é o preço do aumento de produção por hectare $u$, obtido com aplicação da dose $\mathbf{x}_{u}$ de nutriente também por hectare, e $x_{u} t$ é o preço do nutriente aplicado. Logo, sendo $\mathbf{z}=\mathrm{wu} / \mathrm{x}_{\mathrm{u}} \mathrm{t}$, podemos organizar uma tabela para o têrmo

$$
\mathbf{Y}=(1 / \mathrm{c}) \log \mathbf{z}
$$


Fica

$$
\mathbf{x}^{*}=(1 / 2) \mathbf{x}_{\mathbf{u}}+\mathbf{Y},
$$

isto é, para achar a dose econômicamente aconselhável bastará somar o valor $\mathrm{Y}$, obtido da tabela, à metade da dose usada no ensaio de campo.

Damos abajxo duas tabelas, uma para o nitrogênio $(\mathrm{N})$, organizada com $c=0,49$, outra para fósforo $\left(\mathrm{P}_{2} \mathrm{O}_{5}\right)$ e potássio $\left(\mathrm{K}_{2} \mathrm{O}\right)$, preparada com $c=0,88$. Os valores de $c$ adotados se baseiam especialmente nos trabalhos de HODNETT (1956) e PIMENTEL GOMES (1957), mas têm apoio também em numerosas outras informações. Damos também uma terceira tabela, para o estêrco, com $c=0,018 \mathrm{ha} / \mathrm{t}$.

Assim se dados experimentais nos permitirem prever 0 aumento de produção de milho de $600 \mathrm{~kg} / \mathrm{ha}$ para a dose $\mathrm{x}_{\mathrm{u}}$ de $80 \mathrm{~kg} / \mathrm{ha} \mathrm{P}_{2} \mathrm{O}_{5}$, com o milho a Cr $\$ 4,00$ e o nutriente a Cr\$ 10,00 por quilo, teremos:

$$
\mathrm{z}=\frac{600 \times 4}{80 \times 10}=3,00 .
$$

A tabela 2 nos dá $Y=54 \mathrm{~kg} / \mathrm{ha}$, logo a dose econômicamente conveniente é

$$
\mathrm{x}^{*}=80 / 2+54=40+54=94 \mathrm{~kg} / \mathrm{ha} \text { de } \mathrm{P}_{2} \mathrm{O}_{5} .
$$

Se o aumento de produção fôsse $u=200 \mathrm{~kg} / \mathrm{ha}$ para $\mathrm{x}_{\mathrm{u}}=100 \mathrm{~kg} / \mathrm{ha}$ de fósforo $\left(\mathrm{P}_{2} \mathrm{O}_{5}\right)$ então teríamos, aos mesmos preços anteriores, ,

$$
z=\frac{200 \times 4}{100 \times 10}=\frac{800}{1000}=0,8 .
$$

$O$ valor de $Y$, dado pela tabela 2, seria $Y=-11$, logo a dose econômicamente conveniente é

$$
\mathrm{x}^{*}=(1 / 2) 100-11=39 \mathrm{~kg} / \mathrm{ha} \text { de } \mathrm{P}_{2} \mathrm{O}_{5} .
$$

Note-se que quando tivermos $\mathrm{z}=1,0$, isto é, quando o aumento de produção apenas pagar as despêsas de adubação, a dose econômicamente acónselhável será $x^{*}=(1 / 2) x_{u}$, isto é, igual à metade da dose usada no experimento. 
Valores de $\mathrm{Y}$, em $\mathrm{kg} / \mathrm{ha}$, para o nitrogenio (N), calculados com $\mathrm{c}=0,49 \mathrm{ha} /$ quintal métrico.

\begin{tabular}{c|c||c|c||c|c}
\hline $\mathrm{z}$ & $\mathrm{Y}(\mathrm{kg} / \mathrm{ha})$ & $\mathrm{z}$ & $\mathrm{Y}(\mathrm{kg} / \mathrm{ha})$ & $\mathrm{z}$ & $\mathrm{Y}(\mathrm{kg} / \mathrm{ha})$ \\
\hline \hline 0,1 & -204 & 1,6 & 42 & 3,2 & 103 \\
0,2 & -143 & 1,7 & 47 & 3,4 & 108 \\
0,3 & -107 & 1,8 & 52 & 3,6 & 114 \\
0,4 & -81 & 1,9 & 57 & 3,8 & 118 \\
0,5 & -62 & 2,0 & 61 & 4,0 & 123 \\
& -45 & 2,1 & 66 & 4,2 & 127 \\
0,6 & -45 & 2,2 & 70 & 4,4 & 131 \\
0,7 & -32 & 2,3 & 74 & 4,6 & 135 \\
0,8 & -20 & 2,4 & 78 & 4,8 & 139 \\
0,9 & -09 & 81 & 5,0 & 143 \\
1,0 & 00 & 2,5 & 85 & & \\
& & & 8,5 & 151 \\
1,1 & 08 & 2,6 & 85 & 6,0 & 159 \\
1,2 & 16 & 2,7 & 88 & 6,5 & 166 \\
1,3 & 23 & 2,8 & 91 & 7,0 & 172 \\
1,4 & 30 & 2,9 & 94 & 8,0 & 184 \\
1,5 & 36 & 3,0 & 97 & & \\
\hline
\end{tabular}

TABELA 1

Valores de $Y$, em $\mathrm{kg} / \mathrm{ha}$, para o fósforo $\left(\mathrm{P}_{2} \mathrm{O}_{5}\right)$ e o potássio $\left(\mathrm{K}_{2} \mathrm{O}\right)$, calculados com $\mathrm{c}=0,88$ ha/quintal métrico.

\begin{tabular}{c|c||c|c||c|c}
\hline$z$ & $Y(\mathrm{~kg} / \mathrm{ha})$ & $\mathrm{z}$ & $\mathrm{Y}(\mathrm{kg} / \mathrm{ha})$ & $\mathrm{z}$ & $\mathrm{Y}(\mathrm{kg} / \mathrm{ha})$ \\
\hline \hline 0,1 & -114 & 2,1 & 37 & 5,5 & 84 \\
0,2 & -79 & 2,2 & 39 & 6,0 & 88 \\
0,3 & -59 & 2,3 & 41 & 6,5 & 92 \\
0,4 & -45 & 2,4 & 43 & 7,0 & 96 \\
0,5 & --34 & 2,5 & 45 & 7,5 & 99 \\
0,6 & -25 & 2,6 & 47 & 8,0 & 103 \\
0,7 & -18 & 2,7 & 49 & 8,5 & 106 \\
0,8 & -11 & 2,8 & 51 & 9,0 & 108 \\
0,9 & -05 & 2,9 & 52 & 9,5 & 111 \\
1,0 & 00 & 3,0 & 54 & 10,0 & 114 \\
& & & & & \\
1,1 & 05 & 3,2 & 57 & 11,0 & 118 \\
1,2 & 09 & 3,4 & 60 & 12,0 & 123 \\
1,3 & 13 & 3,6 & 63 & 13,0 & 127 \\
1,4 & 17 & 3,8 & 66 & 14,0 & 130 \\
1,5 & 20 & 4,0 & 68 & 15,0 & 134 \\
& & & & 16,0 & 137 \\
1,6 & 23 & 4,2 & 71 & 17,0 & 140 \\
1,7 & 26 & 4,4 & 73 & 18,0 & 143 \\
1,8 & 29 & 4,6 & 75 & 19,0 & 145 \\
1,9 & 32 & 4,8 & 79 & 20,0 & 148 \\
2,0 & 34 & 5,0 & 79 & &
\end{tabular}

TABELA 2 
Valores de $Y$, em $t / h a$, para o estêrco, calculados com $c=0,018 \mathrm{ha} / \mathrm{t}$.

\begin{tabular}{c|c||c|c||c|c}
\hline$z$ & $Y(t / h a)$ & $z$ & $Y$ & $z$ & $Y$ \\
\hline & & & & \\
0,1 & $-55,6$ & 1,6 & 11,3 & 3,2 & 28,1 \\
0,2 & $-38,8$ & 1,7 & 12,8 & 3,4 & 29,5 \\
0,3 & $-29,1$ & 1,8 & 14,2 & 3,6 & 30,9 \\
0,4 & $-22,1$ & 1,9 & 15,5 & 3,8 & 32,2 \\
0,5 & $-16,7$ & 2,0 & 16,7 & 4,0 & 33,4 \\
& & & & & \\
0,6 & $-12,3$ & 2,1 & 18,0 & 4,2 & 34,6 \\
0,7 & $-8,6$ & 2,2 & 19,0 & 4,4 & 35,2 \\
0,8 & $-5,4$ & 2,3 & 20,1 & 4,6 & 36,8 \\
0,9 & $-2,5$ & 2,4 & 21,1 & 4,8 & 37,8 \\
1,0 & 0,0 & 2,5 & 22,1 & 5,0 & 38,8 \\
1,1 & 2,3 & 2,6 & 23,1 & 5,5 & 41,1 \\
1,2 & 4,4 & 2,7 & 24,0 & 6,0 & 43,2 \\
1,3 & 6,3 & 2,8 & 24,8 & 6,5 & 45,2 \\
1,4 & 8,1 & 2,9 & 25,7 & 7,0 & 46,9 \\
1,5 & 9,8 & 3,0 & 26,5 & 8,0 & 50,2 \\
& & & & & \\
\hline
\end{tabular}

TABELA 3

\section{SUMMARY}

The authors discuss a formula for the determination of the most profitable level of fertilization $\left(x^{*}\right)$. This formula, presented by CAREY and ROBINSON (1953), can be written as:

$$
x^{*}=(1 / c) \log \frac{c x_{u} L 10}{1-10^{-c x}}+(1 / c) \log \frac{w u}{x_{u} t}
$$

being $c$ the growth factor in Mitscherlich's equation, $x_{u}$ a standard dressing of the nutrient, $L 10$ the Naeperian logarithm of $10, u$ the response to the standard dressing, $w$ the unit price of the crop product, and $t$ the unit price of the nutrient. This formula is a modification of one of the formulas of PIMENTEL GOMES (1953).

One of its advantages is that is does not depend on A, the theoretical maximum harvest, which is not directly given 
by experimental data. But another advantage, proved in this paper, is that the first term on the right hand side

$$
\mathrm{K}=(1 / \mathrm{c}) \log \frac{\mathrm{cx}_{\mathrm{u}} \mathrm{L} 10}{1-10^{-\mathrm{cx}} \mathrm{u}}
$$

is practically independent of $c$, and approximately equivalent to $(1 / 2) x_{\mathrm{u}}$. So, we have approximately

$$
x^{*}=(1 / 2) x_{u}+(1 / c) \log \frac{w u}{x_{u} t} .
$$

With experimental data we compute

$$
\mathbf{z}=\frac{\mathrm{w} \mathbf{u}}{\mathbf{x}_{\mathrm{u}} \mathrm{t}}
$$

then using tables 1, 2 and 3, we may obtain

and finally

$$
\mathrm{Y}=(1 / \mathrm{c}) \log \mathrm{z}
$$

$$
\mathbf{x}^{*}=(1 / 2) \mathbf{x}_{\mathfrak{u}}+\mathrm{Y} \text {. }
$$

This is an easy way to determine the most profitable level of fertilization when experimental data on the response $u_{4}$ to a dressing $x_{u}$ are avaliable. Tables for the calculation of $Y$ are included, for nitrogen, phosphorus, potash, and manure.

\section{BIBLIOGRAFIA}

CAREY, T. M. \& ROBINSON, 1953 - The Manuring of Sugar Cane. Empire Jour. Exper. Agric. 21: 99-115.

CROWTHER, E. M. \& F. YATES, 1941 - Fertilizer Policy in War-Time. Empire Jour. Exper. Agric. 9: 77-97.

HODNETT, G. R., 1956 - The Response of Sugar Cane to. Fertilizers: Empire Jour. Asper. Agric. 24: 1-19.

PIMENTEL GOMES, F. \& E. MALAVOLTA, 1949 - Aspectos Matemáticos e Estatísticos da Lei de Mitscherlich. Anais da E. S. A. "Luis de Queiroz" 6: 193-229.

PIMENTEL GOMES, F., 1953 - The Use of Mitscherlich's Regression Law in the Analysis of Experiments with Fertilizers. Biometrics 9: 498-516.

PIMENTEL GOMES, F., 1957 - Análise Conjunta de 38 Experimentos de Adubação de Cana-de-Açúcar. Rev. Agricultura 32: 113-126. 